\title{
Expression, Functions, and Treatment Target of PD-L1 (B7-H1) in Multiple Myeloma
}

\author{
Hideto Tamura ${ }^{1 *}$, Mariko Ishibashi ${ }^{2}$, Mika Sunakawa' ${ }^{1}$, Hidemi Takahashi ${ }^{2}$, Koiti Inokuchi $^{1}$ \\ 'Department of Hematology, Nippon Medical School, Tokyo, Japan \\ ${ }^{2}$ Department of Microbiology and Immunology, Nippon Medical School, Tokyo, Japan
}

Article Info

\section{Article Notes}

Received: August 29, 2018

Accepted: October 02, 2018

\section{*Correspondence:}

Dr. Hideto Tamura, Department of Hematology, Nippon Medical School, 1-1-5 Sendagi, Bunkyo-ku, Tokyo 113-8603, Japan; Telephone No: +81-3-3822-2131;

Email:tam@nms.ac.jp.

(c) 2018 Tamura $\mathrm{H}$. This article is distributed under the terms of the Creative Commons Attribution 4.0 International License.

\section{Keywords:}

PD-L1; PD-1

Multiple myeloma

IL-6

PI3K/Akt pathway

\section{ABSTRACT}

Programmed death ligand 1 (PD-L1) expression on myeloma cells is induced by JAK2, STAT3, and MEK1/2-mediated interleukin- 6 signaling, a strong inducer of PD-L1 interferon- $\gamma$ produced by $T$ and natural killer cells, and APRIL produced by osteoclasts in the tumor microenvironment. The soluble form of PD-L1, derived from extracellular domains of PD-L1 molecules expressed in the tumor environment, may also contribute to tumor immune evasion. PD-L1-expressing myeloma cells not only have the ability to escape from the attack of tumor-specific $T$ cells but also high proliferation potential. Furthermore, PD-L1 on myeloma cells delivers a reverse signal to tumor cells through PD-1 binding, resulting in the phosphorylation of Akt accompanied by the acquisition of resistance to anti-myeloma agents. Based on the function of PD-L1 in myeloma, the blockade of the PD-1-PD-L1 pathway is a reasonable treatment in refractory patients. Phase $\mathrm{I} / \mathrm{II}$ clinical trials of anti-PD-1 antibody combined with immunomodulatory drugs demonstrated excellent effects in heavily pretreated multiple myeloma patients with acceptable tolerability. The timing and combination drug of anti-PD-1/PD-L1 antibodies should be considered to improve clinical effects with low mortality in refractory myeloma patients.

\section{Introduction}

Prognosis in patients with multiple myeloma (MM) has improved markedly, although it remains incurable. New treatment strategies including immunotherapies such as immune checkpoint inhibitors and chimeric antigen receptor-T cell (CART) therapy are expected to improve survival rates, and many clinical trials of immunotherapies for MM have been reported and are ongoing ${ }^{1,2}$. For the development of more efficient immunotherapies, it is crucial to elucidate the mechanism of immunosuppression in MM. Programmed death ligand 1 (PD-L1), first identified as a B7 homologue $1(\mathrm{~B} 7-\mathrm{H} 1)$ in humans and mice ${ }^{3,4}$, is expressed on most tumor cells and can induce the apoptosis of tumorspecific T cells, resulting in tumor immune evasion ${ }^{5}$. Immunotherapies targeting the blockade of the PD-1-PD-L1 pathway, which can induce tumor-specific cytotoxic T lymphocytes (CTLs), have shown clinical efficacy in many types of cancer. In hematological malignancies, anti-PD-1 antibodies have been approved for patients with Hodgkin lymphoma, in which high levels of PD-L1 expression on tumor cells occur $^{6}$. This paper reviews the expression and functions of PD-L1 in MM and potential treatment with its inhibitors for refractory MM patients.

PD-L1 Expression in Myeloma Cells and Their
Microenvironment

PD-L1 expression is frequently observed in human cancers. PD-L1 
levels in tumors are regulated in a highly complex manner, with genomic aberrations, transcriptional control, mRNA stability, oncogenic signaling, and protein stability ${ }^{7}$. Kataoka et al. reported a unique genetic mechanism of immune escape caused by structural variations commonly disrupting the 3' region of thePD-L1gene, leading to a marked elevation ofaberrantPD-L1transcripts, in a small subset of patients with hematologic malignancies including adult T-cell leukemia/ lymphoma ${ }^{8}$. Plasma cells from MM patients express higher levels of PD-L1 compared with those from healthy volunteers and patients with monoclonal gammopathy of undetermined significance (MGUS) ${ }^{9,10}$. PD-L1 expression on MM cells is induced by JAK2, STAT3, and MEK1/2-mediated interleukin (IL)- 6 signaling ${ }^{10}$, and a strong inducer of PD-L1 interferon (IFN)- $\gamma$ may be produced by CTLs and natural killer (NK) cells in the tumor microenvironment ${ }^{11}$ (Figure 1). Furthermore, high levels of APRIL produced by osteoclasts increase PDL1 expression on myeloma cells in patients ${ }^{12}$. Some patients with minimal residual disease (MRD) after treatment had higher levels of PD-L1 expression on MM cells compared with the levels before treatment ${ }^{13}$, suggesting that MRD escapes immune surveillance via PD-L1 expression. In the MM microenvironment, immune cells such as myeloid and plasmacytoid dendritic cells (DCs) express higher levels of PD-L1 compared with cells from healthy donors ${ }^{14}$. In MM patients, myeloid-derived suppressor cells (MDSCs), which are a $\mathrm{CD} 11 \mathrm{~b}^{+} \mathrm{CD} 14^{-} \mathrm{HLA}-\mathrm{DR}^{-} \mathrm{CD} 15^{+} \mathrm{CD} 33^{+}$immature myeloid cell population with the ability to suppress innate and adaptive immune responses, are increased in the bone marrow, and some patients were found to have high levels of PD-L1 expressed on MDSCs ${ }^{15,16}$. The expression of the PDL1 receptor PD-1 was detected on NK cells freshly isolated from MM patients but not on those from healthy volunteers ${ }^{17}$. In addition, $\mathrm{PD}-1$ expression on $\mathrm{CD}^{+}$and $\mathrm{CD} 8^{+} \mathrm{T}$ cells was significantly increased in bone marrow samples obtained from MRD-positive and refractory MM patients ${ }^{13}$, and those T cells might be depleted after the interaction with PD-L1. In MM patients, PD-L1 induced in the tumor microenvironment may efficiently induce apoptosis of effector cells for tumor cells, i.e., CTLs and NK cells.

\section{PD-L1 Functions in Myeloma Cells}

PD-L1-expressing MM cells not only have the ability to escape from attack by tumor-specific CTLs but also high proliferation rates and anti-myeloma drug resistance ${ }^{10}$. PD-L $1^{+}$ MM cells express higher levels of Ki-67 and BCL2 compared with PD-L1 ${ }^{-}$MM cells. Consistent with those findings, PD-L1knockdown MM cells had lower proliferation rates and greater percentages of apoptotic cells induced by anti-myeloma agents such as melphalan and bortezomib ${ }^{18}$. We found that PD-L1 on MM cells delivers a reverse signal to tumor cells through PD-1 binding, resulting in phosphorylation of Akt accompanied by the acquisition of drug resistance ${ }^{18}$ (Figure 1), while PD-L1 was reported to deliver anti-apoptotic signals in a mouse tumor model $^{19}$. Our experiments showed that PD-1

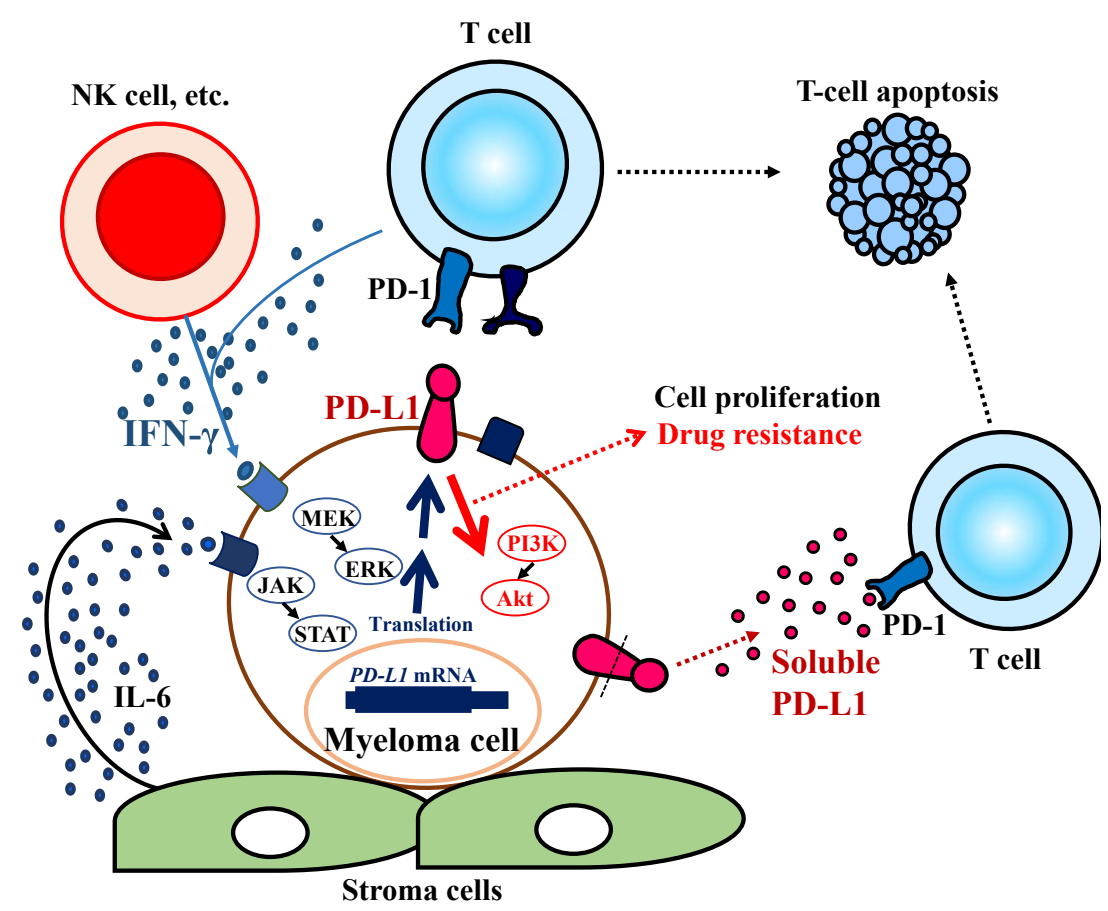

Figure 1: PD-L1 induction and functions in the myeloma microenvironment

PD-L1 expression levels on myeloma cells are upregulated by interleukin (IL)- 6 and IFN- $\gamma$ in the myeloma microenvironment. PD-L1 ${ }^{+}$ myeloma cells have greater potential to proliferate than PD-L1' ${ }^{-}$cells and become resistant to anti-myeloma agents because PD-L1 can deliver PI3K/Akt signals to tumor cells through PD-1 binding. The soluble form of PD-L1 may contribute to escape from immune evasion. 
fusion protein-treated PD-L1-expressing MM cells acquired resistance to anti-myeloma agents such as melphalan and bortezomib with increased BCL2 and decreased FASLG gene expression. Consistent with our results, MRD patient samples showed increased levels of PD-L1 after treatment, and MGUS and asymptomatic $\mathrm{MM}$ patients with $\mathrm{PD}-\mathrm{L} 1^{\text {high }}$ expression on plasma cells developed earlier than those with PD-L1 $1^{\text {low/- }}$ expression $^{20}$.

\section{Soluble Form of PD-L1 in Multiple Myeloma}

The soluble form of PD-L1 (sPD-L1), which is derived from the extracellular domains of PD-L1 molecules expressed on tumor cells, has a molecular weight of $45 \mathrm{kDa}$ and an Ig-V ligand-binding domain that can bind to PD-121 (Figure 1). In vitro analysis showed that SPD-L1 could induce the apoptosis of $\mathrm{T}$ cells ${ }^{22}$. In B-cell lymphoma, patients with higher sPD-L1 levels had shorter survival times compared with those with lower levels ${ }^{23}$. We reported that serum sPD-L1 levels in MM patients were significantly higher than those in healthy volunteers, and high levels of serum sB7-H1 were associated with symptomatic $\mathrm{MM}^{24}$. Furthermore, MM patients with high levels of serum SPD-L1 were found to have a poorer prognosis in terms of progression-free survival and overall survival ${ }^{25}$. However, the mechanism by which the soluble form is shed from the surface of PD-L1 on tumor cells remains unresolved.

\section{Treatment Potential of Anti-PD-1/PD-L1 Antibodies}

Based on the crucial roles of PD-L1 in MM, anti-PD-1 antibody treatment was expected to demonstrate efficacy in MM patients. However, the anti-PD-1 antibody nivolumab alone showed poor clinical results, with stable disease in $63 \%$ of patients ${ }^{1}$; only one of $27 \mathrm{MM}$ patients who responded to nivolumab and achieved stable disease but then experienced disease progression with plasmacytoma achieved a complete response (CR) after radiation therapy for a plasmacytoma of the rib. This suggests that the combination of anti-PD-1 antibody immunotherapy with radiation might be effective in MM, similar to the results in other tumors. The reason for the lack of response to the immune checkpoint inhibitor might be due to the immunosuppression of $\mathrm{T}$ cells because advanced MM patients have various immune deficiencies including insufficient expansion of tumor-specific $\mathrm{CTLs}^{26}$. However, combined immunotherapy with anti-PD-1 antibody plus immunomodulatory drugs (IMiDs) such as lenalidomide and pomalidomide was reported to have surprising effects in heavily pretreated patients. The anti-PD-1 antibody pembrolizumab combined with pomalidomide and dexamethasone was administered to refractory MM patients aged 35-83 (median 64 ) years who had received $2-5$ (median 3 ) prior regimens, including double-refractory patients $(73 \%)$ and patients with high-risk abnormal cytogenetics $(73 \%)$. That phase II clinical trial demonstrated a $60 \%$ overall response rate including a stringent $\mathrm{CR} / \mathrm{CR}$ rate of $8 \%$, very good partial response rate of $19 \%$, and partial response rate of $33 \%$, with a median response duration of 14.7 months ${ }^{27}$. The treatment strategy combining IMiDs with anti-PD-1 antibody was thought to have sufficient clinical efficacy for heavily pretreated MM patients with acceptable tolerability. However, the combination regimen showed an increased mortality rate as detected in the interim analyses of phase III randomized studies, the KEYNOTE-183 (pembrolizumab plus pomalidomide and dexamethasone for relapsed/refractory $\mathrm{MM}$ ) and -185 trials (pembrolizumab in combination with lenalidomide and low-dose dexamethasone in newly diagnosed $\mathrm{MM}$ ), although the increase did not reach statistical significance ${ }^{28}$. The cause of death included cardiac events, which might involve the overactivation of $\mathrm{T}$ cells as seen in PD-1-deficient BALB/c mice that developed autoimmune dilated cardiomyopathy ${ }^{29}$. The response rate was lower in patients who experienced no immune-related adverse events, suggesting that an excess immune response may be associated with high mortality rates. There are no data on anti-PD-L1 antibody treatment in MM, although it might be promising because PD-L1 is expressed not only on MM cells but also on tumor-associated accessory cells in the tumor microenvironment and functions in tumor cells. As further directions, the timing of administration and combination of anti-PD-1/PD-L1 antibodies with other drugs should be considered carefully to improve the clinical outcomes of PD1-PD-L1 pathway blockade in MM patients. Furthermore, to predict the response and occurrence of adverse events, useful markers, for example, levels of PD-L1 expression on MM cells and soluble PD-L1, should be incorporated in future clinical studies using anti-PD-L1/PD-1 antibodies.

\section{Conclusion}

In the MM tumor microenvironment, PD-L1 is inducibly expressed not only on MM cells but also on immune cells including DCs and MDSCs and inhibits the anti-tumor immunity of $\mathrm{T}$ cells. The soluble form of PD-L1 released from tumor cells may induce T-cell apoptosis. Furthermore, PD-L1 on MM cells binding PD-1 can deliver anti-apoptotic signals through the PI3K/Akt signaling pathway, which may be associated with disease progression. The blockade of PD-1 on MM cells increases apoptosis induced by anti-myeloma agents, suggesting the potential of anti-PD-1/PD-L1 antibody treatment combined with chemotherapy. In the future, it will be necessary to assess the timing of the administration of immune checkpoint inhibitors and their combination with other treatment modalities, i.e., radiation, chemotherapy, and T-cell immunotherapies such as DC tumor vaccine and CART therapy.

\section{Acknowledgements}

No financial support or sponsorship was received for the writing of this review.

\section{Conflict of interest}

The authors have no conflict of interest to report. 
Tamura H, Ishibashi M, Sunakawa M, Takahashi H, Inokuchi K. Expression,

\section{References}

1. Lesokhin AM, Ansell SM, Armand P, et al. Nivolumab in patients with relapsed or refractory hematologic malignancy: preliminary results of a phase Ib study. J Clin Oncol. 2016;34:2698-2704.

2. Brudno JN, Maric I, Hartman SD, et al. T cells genetically modified to express an anti-B-cell maturation antigen chimeric antigen receptor cause remissions of poor-prognosis relapsed multiple myeloma. J Clin Oncol. 2018;36:2267-2280.

3. Dong H, Zhu G, Tamada K, et al. B7-H1, a third member of the B7 family, co-stimulates T-cell proliferation and interleukin-10 secretion. Nat Med. 1999;5:1365-1369.

4. Tamura H, Dong H, Zhu G, et al. B7-H1 costimulation preferentially enhances CD28-independent T-helper cell function. Blood 2001;97:1809-1816.

5. Dong H, Strome SE, Salomao DR, et al. Tumor-associated B7-H1 promotes T-cell apoptosis: a potential mechanism of immune evasion. Nat Med. 2002;8:793-800.

6. Ansell SM, Lesokhin AM, Borrello I, et al. PD-1 blockade with nivolumab in relapsed or refractory Hodgkin's lymphoma. N Engl J Med. 2015;372;311-319.

7. Sun C, Mezzadra R, Schumacher TN. Regulation and function of the PD-L1 checkpoint. Immunity. 2018;48:434-452.

8. Kataoka K,ShiraishiY, TakedaY,etal.AberrantPD-L1 expressionthrough 3'-UTR disruption in multiple cancers.Nature.2016;534:402-406.

9. Liu J, Hamrouni A, Wolowiec D, et al. Plasma cells from multiple myeloma patients express B7-H1 (PD-L1) and increase expression after stimulation with IFN- $\gamma$ and TLR ligands via a MyD88-, TRAF6-, and MEK-dependent pathway. Blood. 2007;110:296-304.

10. Tamura H, Ishibashi M, Yamashita T, et al. Marrow stromal cells induce B7-H1 expression on myeloma cells, generating aggressive characteristics in multiple myeloma. Leukemia. 2013;27:464-472.

11. Lee SJ, Jang BC, Lee SW, et al. Interferon regulatory factor- 1 is prerequisite to the constitutive expression and IFN-gamma-induced upregulation of B7-H1 (CD274). FEBS Lett. 2006;580:755-762.

12. An G, Acharya C, Feng X, et al. Osteoclasts promote immune suppressive microenvironment in multiple myeloma: therapeutic implication. Blood. 2016;128:1590-1603.

13. Paiva B, Azpilikueta A, Puig N, et al. PD-L1/PD-1 presence in the tumor microenvironment and activity of PD-1 blockade in multiple myeloma. Leukemia. 2015;29:2110-2113.

14. Sponaas AM, Moharrami NN, Feyzi E, et al. PDL1 expression on plasma and dendritic cells in myeloma bone marrow suggests benefit of targeted anti-PD1-PDL1 therapy. PLoS One. 2015;10:e0139867.

15. Görgun G, Samur MK, Cowens KB, et al. Lenalidomide enhances immune checkpoint blockade-induced immune response in multiple myeloma. Clin Cancer Res. 2015;21:4607-4618.

16. Malek E, de Lima M, Letterio JJ, et al. Myeloid-derived suppressor cells: The green light for myeloma immune escape. Blood Rev. 2016;30:341-348.

17. Benson DM Jr, Bakan CE, Mishra A, et al. The PD-1/PD-L1 axis modulates the natural killer cell versus multiple myeloma effect: a therapeutic target for CT-011, a novel monoclonal anti-PD-1 antibody. Blood. 2010;116:2286-2294.

18. Ishibashi $M$, Tamura $H$, Sunakawa $M$, et al. Myeloma drug resistance induced by binding of myeloma B7-H1 (PD-L1) to PD-1. Cancer Immunol Res. 2016;4:779-788.

19. Azuma T, Yao S, Zhu G, et al. B7-H1is a ubiquitous antiapoptotic receptor on cancer cells. Blood. 2008;111:3635-3643.

20. Dhodapkar MV, Sexton R, Das R, et al. Prospective analysis of antigenspecific immunity, stem-cell antigens, and immune checkpoints in monoclonal gammopathy. Blood. 2015;126:2475-2478.

21. Frigola X, Inman BA, Lohse CM, et al. Identification of a soluble form of B7-H1 that retains immunosuppressive activity and is associated with aggressive renal cell carcinoma. Clin Cancer Res. 2011;17:1915-1923.

22. Frigola X, Inman BA, Krco CJ, et al. Soluble B7-H1: differences in production between dendritic cells and $\mathrm{T}$ cells. Immunol Lett. 2012;142:78-82.

23. Rossille D, Gressier M, Damotte D, et al. High level of soluble programmed cell death ligand 1 in blood impacts overall survival in aggressive diffuse large B-cell lymphoma: results from a French multicenter clinical trial. Leukemia. 2014;28:2367-2375.

24. Sunakawa $M$, Tamura $H$, Ishibashi $M$, et al. Clinical impact and possible immunosuppressive function of soluble B7-H1 (PD-L1) in multiple myeloma. Clin Lymphoma Myeloma Leuk. 2017;17(Suppl):e110e111.

25. Wang L, Wang $\mathrm{H}$, Chen $\mathrm{H}$, et al. Serum levels of soluble programmed death ligand 1 predict treatment response and progression free survival in multiple myeloma. Oncotarget. 2015;6:41228-41236.

26. Tamura H. Immunopathogenesis and immunotherapy of multiple myeloma. Int J Hematol. 2018;107:278-285.

27. Badros A, Hyjek E, Ma N, et al. Pembrolizumab, pomalidomide, and low-dose dexamethasone for relapsed/refractory multiple myeloma. Blood. 2017;130:1189-1197.

28. Krauss AC, Mulkey F, Shen YL, et al. FDA analysis of pembrolizumab trials in multiple myeloma: immune related adverse events and response. J Clin Oncol. 2018;36(suppl):abstr 8008.

29. Nishimura H, Okazaki T, Tanaka $Y$, et al.Autoimmune dilated cardiomyopathy in PD-1 receptor-deficient mice. Science. 2001;291:319-322. 